\title{
Prevalence and Prognostic Significance of Functional Mitral and Tricuspid Regurgitation Despite Preserved Left Ventricular Ejection Fraction in Atrial Fibrillation Patients
}

\author{
Yukio Abe, MD; Kanako Akamatsu, MD; Kazato Ito, MD; Yoshiki Matsumura, MD, PhD; \\ Kenji Shimeno, MD, PhD; Takahiko Naruko, MD, PhD; Yosuke Takahashi, MD, PhD; \\ Toshihiko Shibata, MD, PhD; Minoru Yoshiyama, MD, PhD
}

\begin{abstract}
Background: We investigated the prevalence and prognostic significance of functional mitral regurgitation (MR) and tricuspid regurgitation (TR) in patients with atrial fibrillation (AF) and preserved left ventricular ejection fraction (LVEF).

Methods and Results: We retrospectively studied the cases of 11,021 consecutive patients who had undergone transthoracic echocardiography. AF appeared in 1,194 patients, and we selected 298 with AF and LVEF $\geq 50 \%$ but without other underlying heart diseases. Moderate or greater (significant) degree of functional MR and of TR was seen in $24(8.1 \%)$ and in $44(15 \%)$ patients, respectively $(P=0.0045)$. In contrast, significant MR and TR were more frequently seen in patients with AF duration $>10$ years $(28 \%$ vs. $25 \%$, respectively). During the follow-up period of $24 \pm 17$ months, 35 patients (12\%) met the composite endpoint defined as cardiac death, admission due to heart failure, or mitral and/or tricuspid valve surgery. On Cox proportional hazard ratio analysis, both MR and TR grading predicted the endpoint, independently of other echocardiographic parameters. On Kaplan-Meyer analysis, presence of both significant functional MR and TR was associated with poor prognosis, with an event-free rate of only $21 \%$ at the mean follow-up period of 24 months.
\end{abstract}

Conclusions: Significant functional MR and TR are seen in a substantial proportion of patients with longstanding AF, despite preserved LVEF. This MR/TR combination predicts poor outcome for AF patients, who may have to be treated more intensively.

Key Words: Atrial fibrillation; Echocardiography; Heart failure; Mitral regurgitation; Tricuspid regurgitation

$\mathbf{T}$ he incidence of atrial fibrillation (AF) increases with age, ${ }^{1-3}$ and heart failure (HF) is the most important cause of mortality in elderly AF patients. ${ }^{46} \mathrm{AF}$ can develop after HF, but it can also precede HF with reduced left ventricular ejection fraction (LVEF) or HF with preserved LVEF. ${ }^{\text {-9 }}$ Patients with AF and subsequent HF have a poor prognosis, but the determinants that generate $\mathrm{HF}$ in AF patients are still uncertain.

Secondary (functional) mitral regurgitation (MR) and tricuspid regurgitation (TR) can occur as a result of atrial dilatation in AF patients, despite having preserved left ventricular (LV) systolic function. 10-16 These can be termed "atrial functional MR" and "atrial functional TR," respectively. Both atrial functional MR and TR resulting from atrial dilatation have recently received much attention, but little information is available regarding the disease characteristics of these valvular regurgitations and their impacts on HF. In addition, very few descriptions are available for the MR and TR secondary to AF in the current guidelines for valvular heart disease. ${ }^{17,18}$ The aim of the present retro- spective analysis was therefore to investigate the prevalence and the prognostic significance of the occurrence of functional MR and TR despite preserved LV systolic function in patients with AF.

\section{Methods}

\section{Subjects and Data Collection}

We retrospectively analyzed the echocardiography laboratory database and the medical records at Osaka City General Hospital, Osaka, Japan. We selected patients with $\mathrm{AF}$ and preserved $\mathrm{LVEF}(\geq 50 \%)$ who underwent transthoracic echocardiography (TTE) in the echocardiography laboratory over a period of 2 consecutive years (June 2012-May 2014). We excluded patients with acute decompensated HF (ADHF), a moderate or greater degree of organic valvular heart disease, apparent degenerative changes in mitral or tricuspid valve complex, a history of coronary artery disease or regional LV wall motion abnormality suggesting myocardial ischemia or infarction, or a

Received December 4, 2017; revised manuscript received January 15, 2018; accepted January 29, 2018; released online March 16, 2018 Time for primary review: 21 days

Department of Cardiology, Osaka City General Hospital, Osaka (Y.A., K.A., K.I., Y.M., K.S., T.N.); Department of Cardiovascular Surgery (Y.T., T.S.), Department of Cardiology (M.Y.), Osaka City University Medical School, Osaka, Japan

Mailing address: Yukio Abe, MD, Department of Cardiology, Osaka City General Hospital, 2-13-22 Miyakojima-hondori, Miyakojima-ku, Osaka 534-0021, Japan. E-mail: abeyukio@aol.com

ISSN-1346-9843 All rights are reserved to the Japanese Circulation Society. For permissions, please e-mail: cj@j-circ.or.jp 
Table 1. AF Patients With Preserved LVEF: Subject Characteristics vs. MR and TR Status

\begin{tabular}{|c|c|c|c|c|c|}
\hline & \multicolumn{4}{|c|}{ Significant ( $\geq$ moderate) MR and TR } & \multirow{2}{*}{$\begin{array}{l}\text { A prior } \\
\text { P-value }\end{array}$} \\
\hline & $\begin{array}{c}\text { Neither MR nor TR } \\
(n=241)\end{array}$ & $\begin{array}{c}\text { Only TR } \\
(n=33)\end{array}$ & $\begin{array}{c}\text { Only MR } \\
(n=13)\end{array}$ & $\begin{array}{c}\text { Both MR and TR } \\
(n=11)\end{array}$ & \\
\hline Age (years) & $71 \pm 10$ & $78 \pm 9^{*}, \dagger$ & $69 \pm 13^{*}, \ddagger$ & $80 \pm 7^{\star,+, \S}$ & $<0.001$ \\
\hline Men & $155(64)$ & $12(36)^{\star}, \dagger$ & $6(46)$ & $5(45)$ & 0.009 \\
\hline NYHA functional class & $1.2 \pm 0.4$ & $1.5 \pm 0.6^{*, \dagger}$ & $1.6 \pm 0.8^{*, \dagger}$ & $1.8 \pm 0.6^{\star}, \dagger$ & $<0.001$ \\
\hline Prior HF admission & $27(11)$ & $7(21)$ & $6(46)^{\star}, \dagger$ & $7(64)^{*,+, \ddagger}$ & $<0.001$ \\
\hline Hypertension & $125(52)$ & $17(52)$ & $7(54)$ & $5(45)$ & 0.98 \\
\hline Diabetes mellitus & $68(28)$ & $4(12)$ & $1(8)$ & $1(9)$ & 0.050 \\
\hline Dyslipidemia & $71(29)$ & $10(30)$ & $1(8)$ & $2(18)$ & 0.32 \\
\hline Digitalis & $61(25)$ & $15(45)$ & $6(46)$ & $5(45)$ & 0.030 \\
\hline ACEI or ARB & $91(38)$ & $11(33)$ & $5(38)$ & $7(64)$ & 0.35 \\
\hline$\beta$-blocker & $86(36)$ & $11(33)$ & $7(54)$ & $4(36)$ & 0.60 \\
\hline Aldosterone antagonist & $32(13)$ & $10(30)$ & $2(15)$ & $5(45)$ & 0.005 \\
\hline Other diuretics & $78(32)$ & $17(52)$ & $9(69)^{\star}, \dagger$ & $8(73)^{\star}, \dagger$ & $<0.001$ \\
\hline Anticoagulant agents & $170(70)$ & $27(82)$ & $10(77)$ & $10(91)$ & 0.25 \\
\hline Statins & $48(20)$ & $9(27)$ & $0(0)$ & $0(0)$ & 0.067 \\
\hline
\end{tabular}

Data given as mean \pm SD or $n(\%)$. ${ }^{*}<<0.05$ vs. ${ }^{\dagger}$ patients with neither MR nor TR, ‡patients with only TR, or \$patients with only MR (post-hoc test). ACEI, angiotensin-converting enzyme inhibitor; AF, atrial fibrillation; ARB, angiotensin receptor blocker; HF, heart failure; LVEF, left ventricular ejection fraction; MR, mitral regurgitation; NYHA, New York Heart Association; TR, tricuspid regurgitation.

definite diagnosis of cardiomyopathy. Patients who had undergone cardiac device implantation or cardiac surgery, patients with AF spontaneously or therapeutically defibrillated within 1 week after TTE, and patients with $<1$ week of follow-up data were also excluded. In patients who underwent repeated TTE for the 2 years, the earliest TTE was considered as the entry point into the study, and the repeated TTE were excluded.

The clinical, electrocardiographic (ECG), and echocardiographic data at TTE were retrospectively collected. Clinical data included demographics, presenting symptoms, cardiovascular risk factors, and medical treatment at TTE. The clinical information parameters are listed in Table $\mathbf{1 .}$ The duration of AF was estimated if the ECG data or other specific information in the medical record were available. The echocardiographic data were collected from the TTE database. The occurrence of cardiac events after TTE was also recorded, and the primary composite endpoint was defined as cardiac death that included sudden death with an unknown cause, hospitalization due to worsening HF, or mitral valve and/or tricuspid valve repair. According to the consensus of the Department of Cardiology and Department of Cardiovascular Surgery, Osaka City General Hospital, we usually performed mitral valve repair and concomitant tricuspid valve repair in patients who had permanent $\mathrm{AF}$ that had persisted for $>1$ year, chronic moderate or severe MR with at least mild TR, chronic HF symptoms of at least New York Heart Association (NYHA) functional class II, and at least 1 prior admission for ADHF complicated by severe MR. ${ }^{12}$

This study was approved by the institutional review board, with a waiver of individual consent.

\section{Echocardiography}

All TTE was performed by expert level 3 sonographers according to the American Society of Echocardiography definition, ${ }^{19}$ and the results were interpreted by experienced attending doctors in the echocardiography laboratory. Any disagreements were resolved on consensus reading.
For all measurements of systolic parameters in AF patients, 1 beat occurring after 2 serial beats with average RR interval was carefully selected. ${ }^{20}$ For all measurements of diastolic Doppler parameters in AF patients, 1 beat with an average Doppler-wave contour with an average velocity was visually, but carefully, selected. The LV end-diastolic dimension, LV end-systolic dimension, LVEF, LV mass, and left atrial (LA) dimension were measured according to the guidelines. ${ }^{21}$ We indexed these parameters by dividing them by the body surface area. The severity of the MR was defined using a multiparametric approach, including an assessment of the color Doppler-derived jet area, the effective regurgitant orifice area using the proximal isovelocity surface area method, the MR volume and fraction using the Doppler-derived volumetric method, and the pulmonary vein flow velocity pattern. ${ }^{22} \mathrm{TR}$ grade was also defined using a multiparametric approach, including an assessment of the color Doppler-derived jet area, the continuous wave Doppler-derived jet density and contour, and the hepatic vein flow velocity pattern. ${ }^{22}$ The methods for the assessments of these valvular regurgitations were selected per patient under the careful consideration of the methodological advantages and limitations, according to the American Society of Echocardiography guidelines. ${ }^{22}$ MR and TR were graded as none, mild, moderate, or severe. When a considerable discrepancy was evident in the grading between the multiple methods or between the different beats in AF patients, we used the following 3 levels of borderline grading: none-mild; mild-moderate; and moderate-severe. For the statistical analysis, the grading was scored as follows: none, 0 ; none-mild, 0.5 ; mild, 1 ; mild-moderate, 1.5; moderate, 2; moderate-severe, 2.5; and severe, 3. A $\geq$ moderate degree of MR or TR (i.e., 2, 2.5, or 3 ) was defined as significant in the present study. Continuous wave Doppler was used to obtain the TR peak velocity (v, $\mathrm{m} / \mathrm{s}$ ) and the transtricuspid systolic pressure gradient $(\mathrm{mmHg})$, which was calculated as $4 \times \mathrm{v}^{2}$. The right ventricular systolic pressure (RVSP) was then estimated as the sum of the estimated transtricuspid systolic pressure gradient and 


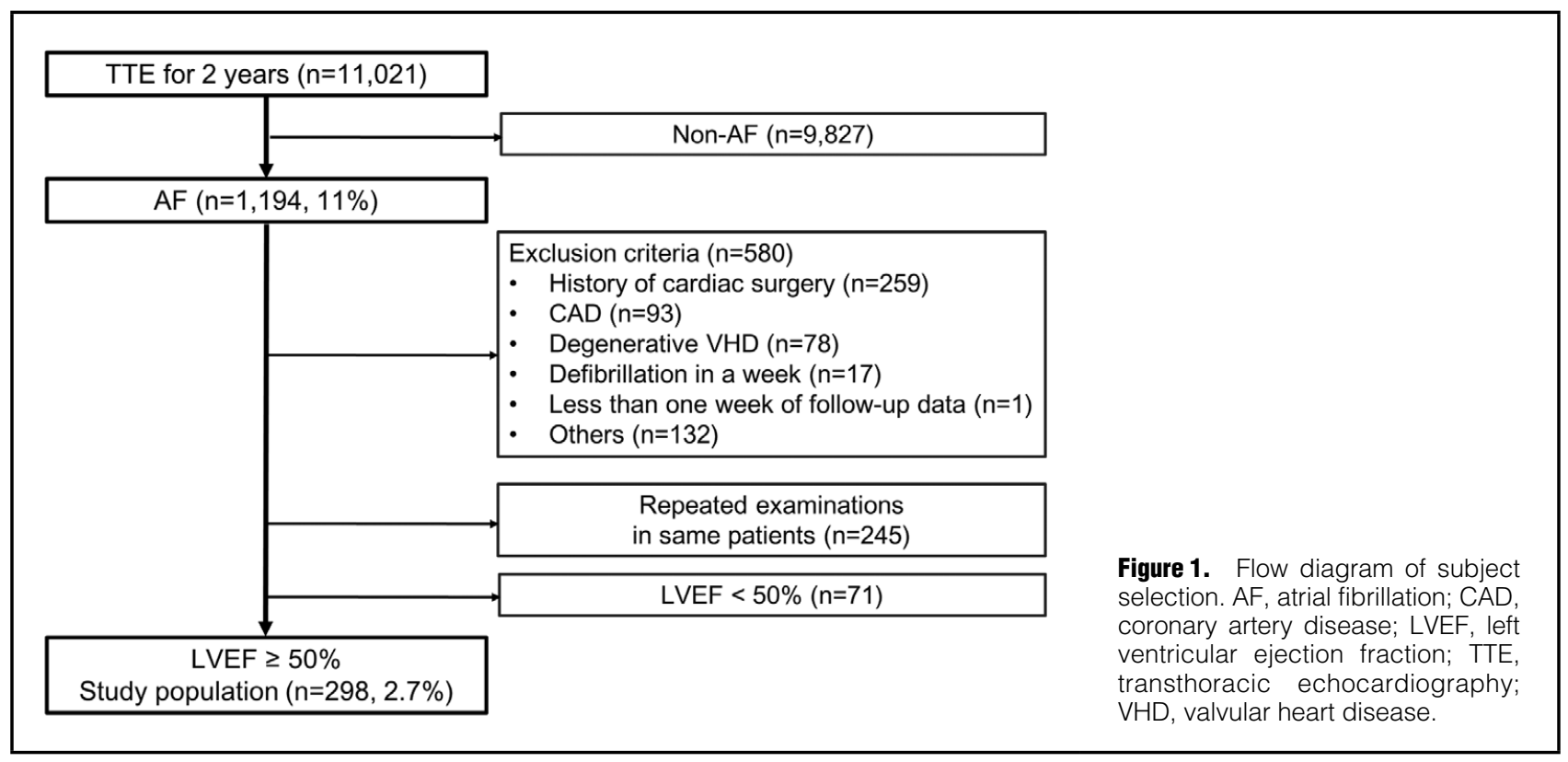

right atrial (RA) pressure. The RA pressure was estimated as follows: an inferior vena cava (IVC) diameter $\leq 2.1 \mathrm{~cm}$ that collapsed by $\geq 50 \%$ with a sniff was considered to indicate a normal RA pressure of $3 \mathrm{mmHg}$, whereas an IVC diameter $>2.1 \mathrm{~cm}$ that collapsed $<50 \%$ with a sniff was considered to indicate a high RA pressure of $15 \mathrm{mmHg}$. In scenarios in which the IVC diameter and collapse did not fit this paradigm, an intermediate value of $8 \mathrm{mmHg}$ was assigned. ${ }^{23}$ The transmitral flow velocity curves in diastole and the mitral annular tissue Doppler imaging signals were obtained as previously described. ${ }^{24}$ The peak velocity of early diastolic flow across the mitral valve (E) and the deceleration time were measured. The peak early diastolic tissue Doppler velocity of the medial mitral annulus (e') on apical 4-chamber view was measured, and the E/e' ratio was calculated.

\section{Statistical Analysis}

The categorical variables are expressed as absolute values and percentages and were compared using chi-squared test, McNemar test, or Kruskal-Wallis test followed by posthoc pairwise test. The continuous variables are expressed as mean \pm SD and were compared using 1-way analysis of variance followed by the post-hoc Tukey-Kramer test, or Kruskal-Wallis test followed by the post-hoc pairwise test. The significant predictors of the primary endpoint were identified on univariate Cox proportional hazards regression analysis. We then performed a multivariate Cox proportional hazards regression analysis based on stepwise selection with a model using significant clinical predictors and a model using significant echocardiographic predictors to determine the independent predictors of the primary endpoint. The Kaplan-Meier method was used to evaluate event-free survival. Statistical analysis was performed using MedCalc (version 15.8, MedCalc Software, Ostend, Belgium). $\mathrm{P}<0.05$ was considered statistically significant.

\section{Results}

Subject selection is shown in Figure 1. Of a total of 11,021 patients who underwent TTE in the echocardiography laboratory for the 2 years, 1,194 (11\%) had AF at TTE. We excluded 580 patients who met the exclusion criteria and 245 TTE repeated in the same patients. Of the remaining patients, 298 (2.7\% of the total cohort) had LVEF $\geq 50 \%$, and constituted the final study cohort. All data for the clinical and echocardiographic parameters were collected with missing values $<5 \%$, except for AF duration, E/e', and estimated RVSP, which had missing values of $56 \%, 6 \%$ and $24 \%$, respectively. Therefore, we excluded AF duration, E/e', and estimated RVSP from the subsequent Cox hazard proportional hazards regression analysis.

Of the 298 patients, $241(81 \%)$ were referred from the outpatient departments. The remaining $57(19 \%)$ were referred from the wards, whereas all of them had stable conditions before discharge. The mean age of the 298 patients was $72 \pm 10$ years, and $178(60 \%)$ were male. In these patients, significant MR and TR, defined as $\geq$ moderate MR and TR, were seen in $24(8.1 \%)$ patients and in 44 $(15 \%)$ patients, respectively $(\mathrm{P}=0.0045$; Figure 2$)$. Only 11 (3.7\%) had both significant MR and significant TR.

Patient characteristics according to significant MR and TR status are listed in Table 1. The patients with both significant MR and significant TR had the highest age ( $80 \pm 7$ years old), the highest NYHA functional class $(1.8 \pm 0.6)$, and the most frequent history of prior admission due to HF (64\%). The echocardiographic data according to significant MR and TR status are listed in Table 2 . The patients with both significant MR and significant TR had the largest $\mathrm{LV}$ diastolic dimension index $\left(35 \pm 5 \mathrm{~mm} / \mathrm{m}^{2}\right)$, LV systolic dimension index $\left(23 \pm 5 \mathrm{~mm} / \mathrm{m}^{2}\right)$, LA dimension index $\left(37 \pm 8 \mathrm{~mm} / \mathrm{m}^{2}\right)$, and estimated RVSP $(42 \pm 12 \mathrm{mmHg})$. In contrast, LVEF was similar regardless of the presence or absence of significant MR and TR.

The duration of AF was retrospectively confirmed on ECG or on the description in the medical records in 131 $(44 \%)$ of the 298 patients. The relationship between AF duration and the prevalence of significant ( $\geq$ moderate) MR or TR is shown in Figure 3. Of the patients with AF duration $\leq 1$ year, $3 \%$ and none had significant $\mathrm{MR}$ and 
significant TR, respectively. In contrast, of the patients with AF duration $>10$ years, approximately one-fourth (28\% and $25 \%$ ) had significant MR and significant TR, respectively.

During the follow-up period of $24 \pm 17$ months (median, 32 months; range, $0.3-54$ months), 35 patients (12\%) met the primary endpoint, consisting of 5 cardiac deaths, 23 hospitalizations due to ADHF, and 7 mitral valve and tricuspid valve repairs. In contrast, only 7 patients $(2.3 \%)$ had cerebral infarction, and only $4(1.3 \%)$ had major hemorrhagic events, including 3 intracranial hemorrhages and 1 traumatic intramuscular hematoma at the shoulder.

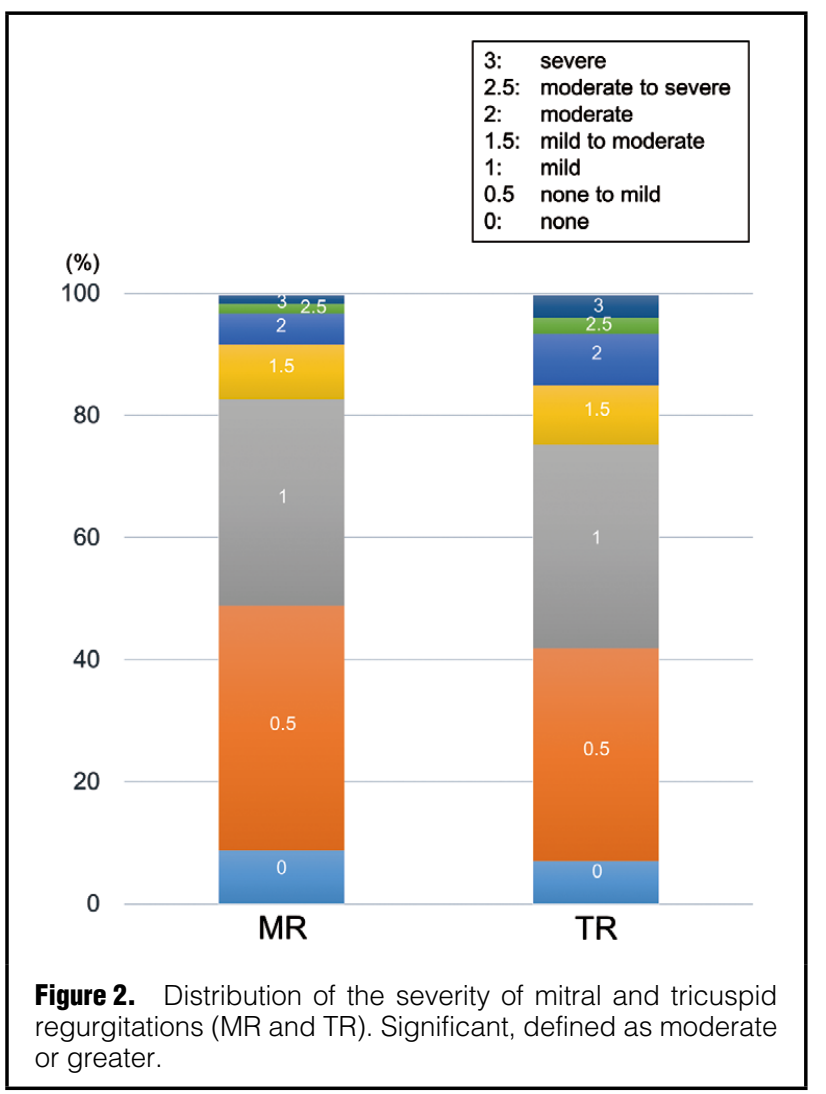

Table 3 lists the results of the Cox proportional hazards regression analysis to identify the predictors of the primary endpoint. In the model using clinical data, the factors of age, NYHA functional class, history of prior admission due to HF, and absence of dyslipidemia could independently predict the endpoint. In the model using echocardiographic data, both the MR grade and the TR grade could predict the endpoint independently of each other and independently of other echocardiographic parameters. The hazard ratios per 1-grade increase in MR and TR were 4.0 (95\% CI: $2.3-7.0)$ and 1.8 (95\% CI: 1.1-2.9), respectively.

On Kaplan-Meyer analysis, patients with significant functional MR had poorer prognosis than patients without significant functional MR: the event-free rate was 39\% with the standard error of $11 \%$, vs. $94 \%$ with the standard error of $2 \%$, respectively, at mean follow-up of 24 months $(\log$ rank $\mathrm{P}<0.0001$; Figure 4A). As well, the patients with significant functional TR had poorer prognosis than patients without significant functional TR: the event-free

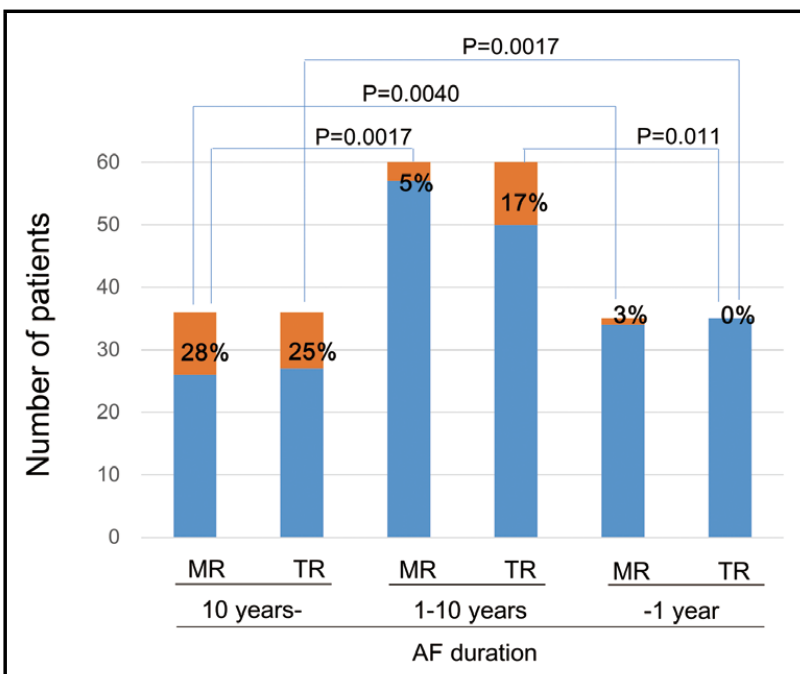

Figure 3. Prevalence of significant (i.e., moderate or greater) mitral or tricuspid regurgitation (MR or TR) vs. duration of atrial fibrillation (AF). Blue, not significant; orange, significant.

\begin{tabular}{|c|c|c|c|c|c|}
\hline & \multicolumn{4}{|c|}{ Significant ( $\geq$ moderate) MR and TR } & \multirow{2}{*}{$\begin{array}{l}\text { A prior } \\
\text { P-value }\end{array}$} \\
\hline & $\begin{array}{c}\text { Neither MR nor TR } \\
(n=241)\end{array}$ & $\begin{array}{c}\text { Only TR } \\
(n=33)\end{array}$ & $\begin{array}{c}\text { Only MR } \\
(n=13)\end{array}$ & $\begin{array}{l}\text { Both MR and TR } \\
(n=11)\end{array}$ & \\
\hline LV mass index $\left(\mathrm{g} / \mathrm{m}^{2}\right)$ & $88 \pm 23$ & $88 \pm 19$ & $122 \pm 56^{*}, \mathrm{t}, \ddagger$ & $116 \pm 38^{*,+, \neq}$ & 0.007 \\
\hline LVDd index $\left(\mathrm{mm} / \mathrm{m}^{2}\right)$ & $27 \pm 4$ & $29 \pm 4^{*}, \dagger$ & $33 \pm 5^{\star}, t, \neq$ & $35 \pm 5^{\star, t, \neq}$ & $<0.001$ \\
\hline LVDs index $\left(\mathrm{mm} / \mathrm{m}^{2}\right)$ & $17 \pm 3$ & $19 \pm 3^{*, \dagger}$ & $21 \pm 4^{*, \dagger}$ & $23 \pm 5^{\star},+, \neq$ & $<0.001$ \\
\hline LVEF (\%) & $66 \pm 7$ & $64 \pm 8$ & $64 \pm 6$ & $64 \pm 7$ & 0.49 \\
\hline LA dimension index $\left(\mathrm{mm} / \mathrm{m}^{2}\right)$ & $26 \pm 5$ & $30 \pm 6^{*, \dagger}$ & $35 \pm 5^{*, t, \neq}$ & $37 \pm 8^{*,+, \neq}$ & $<0.001$ \\
\hline E wave velocity $(\mathrm{cm} / \mathrm{s})$ & $85 \pm 20$ & $96 \pm 28^{*, \dagger}$ & $117 \pm 41^{*, \dagger}$ & $110 \pm 24^{*}, \dagger$ & $<0.001$ \\
\hline $\mathrm{DT}(\mathrm{ms})$ & $179 \pm 47$ & $174 \pm 37$ & $175 \pm 47$ & $171 \pm 58$ & 0.90 \\
\hline $\mathrm{e}^{\prime}(\mathrm{cm} / \mathrm{s})$ & $8.2 \pm 2.4$ & $7.9 \pm 2.1$ & $7.3 \pm 2.8$ & $8.6 \pm 2.1$ & 0.60 \\
\hline$E / e^{\prime}$ & $11.4 \pm 4.7$ & $12.9 \pm 6.2$ & $17.3 \pm 6.9^{*}, \dagger$ & $13.6 \pm 5.0$ & 0.001 \\
\hline Estimated RVSP (mmHg) & $28 \pm 7$ & $37 \pm 13^{*, \dagger}$ & $34 \pm 10^{\star}, \dagger$ & $42 \pm 12^{*, \dagger}$ & $<0.001$ \\
\hline
\end{tabular}

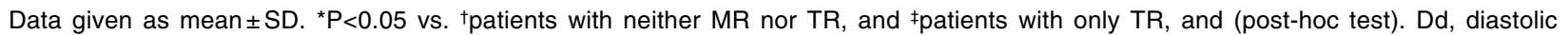
dimension; Ds, systolic dimension; DT, deceleration time; LA, left atrial; LV, left ventricular; RVSP, right ventricular systolic pressure. Other abbreviations as in Table 1. 
Table 3. AF Patients With Preserved LVEF: Significant Predictors of the Primary Endpoint ${ }^{\dagger}$

\begin{tabular}{|c|c|c|c|c|c|c|}
\hline & \multicolumn{3}{|c|}{ Univariate analysis } & \multicolumn{3}{|c|}{ Multivariate analysis } \\
\hline & HR & $95 \% \mathrm{Cl}$ & P-value & HR & $95 \% \mathrm{Cl}$ & P-value \\
\hline \multicolumn{7}{|l|}{ Model using clinical data } \\
\hline Age (per 10-year increase) & 2.2 & $1.4-3.5$ & 0.0005 & 1.8 & $1.1-2.9$ & 0.011 \\
\hline NYHA functional class & 3.0 & $1.8-4.8$ & $<0.0001$ & 2.2 & $1.4-3.6$ & 0.0015 \\
\hline Prior HF admission & 4.4 & $2.3-8.6$ & $<0.0001$ & 4.1 & $2.1-8.2$ & $<0.0001$ \\
\hline Dyslipidemia & 0.31 & $0.11-0.88$ & 0.027 & 0.32 & $0.11-0.92$ & 0.034 \\
\hline Aldosterone antagonist & 2.1 & $1.02-4.5$ & 0.043 & - & - & - \\
\hline Other diuretics & 2.3 & $1.2-4.6$ & 0.015 & - & - & - \\
\hline \multicolumn{7}{|l|}{ Model using echocardiographic data } \\
\hline LV mass index $\left(\mathrm{g} / \mathrm{m}^{2}\right)$ (per $10-\mathrm{g} / \mathrm{m}^{2}$ increase) & 1.2 & $1.1-1.3$ & $<0.0001$ & - & - & - \\
\hline LVDd index $\left(\mathrm{mm} / \mathrm{m}^{2}\right)$ & 1.2 & $1.1-1.3$ & $<0.0001$ & - & - & - \\
\hline LVDs index $\left(\mathrm{mm} / \mathrm{m}^{2}\right)$ & 1.2 & $1.1-1.3$ & $<0.0001$ & - & - & - \\
\hline LA dimension index $\left(\mathrm{mm} / \mathrm{m}^{2}\right)$ & 1.13 & $1.09-1.2$ & $<0.0001$ & - & - & - \\
\hline MR grade (per 1-grade increase) & 5.0 & $3.3-7.5$ & $<0.0001$ & 4.0 & $2.3-7.0$ & $<0.0001$ \\
\hline TR grade (per 1-grade increase) & 3.3 & $2.3-4.8$ & $<0.0001$ & 1.8 & $1.1-2.9$ & 0.0012 \\
\hline E wave velocity $(\mathrm{cm} / \mathrm{s})$ (per 10-cm/s increase) & 1.3 & $1.2-1.5$ & $<0.0001$ & - & - & - \\
\hline
\end{tabular}

${ }^{\dagger}$ Cardiac death, hospitalization due to worsening heart failure, or mitral valve and/or tricuspid valve repair. Abbreviations as in Tables 1,2 .

A

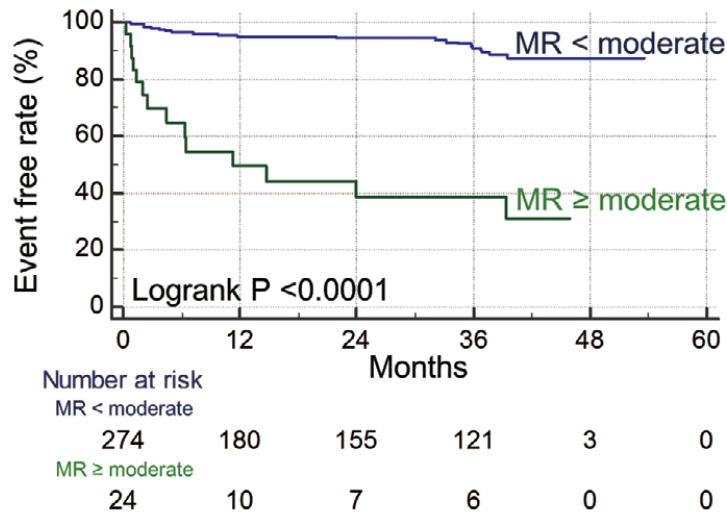

C

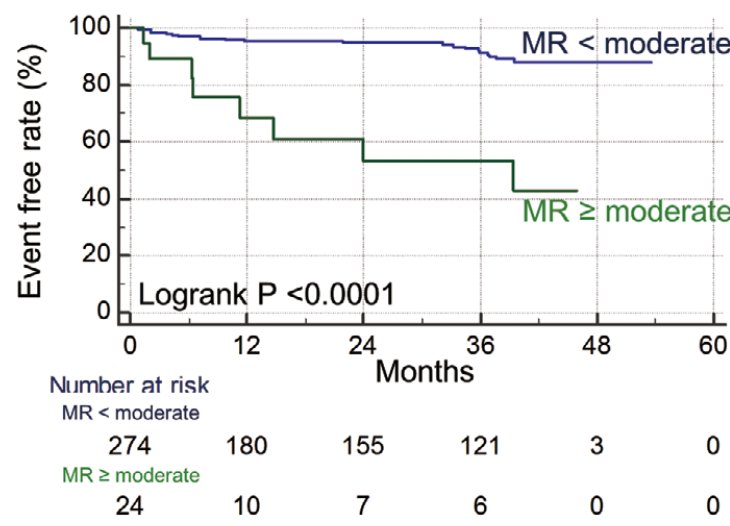

B
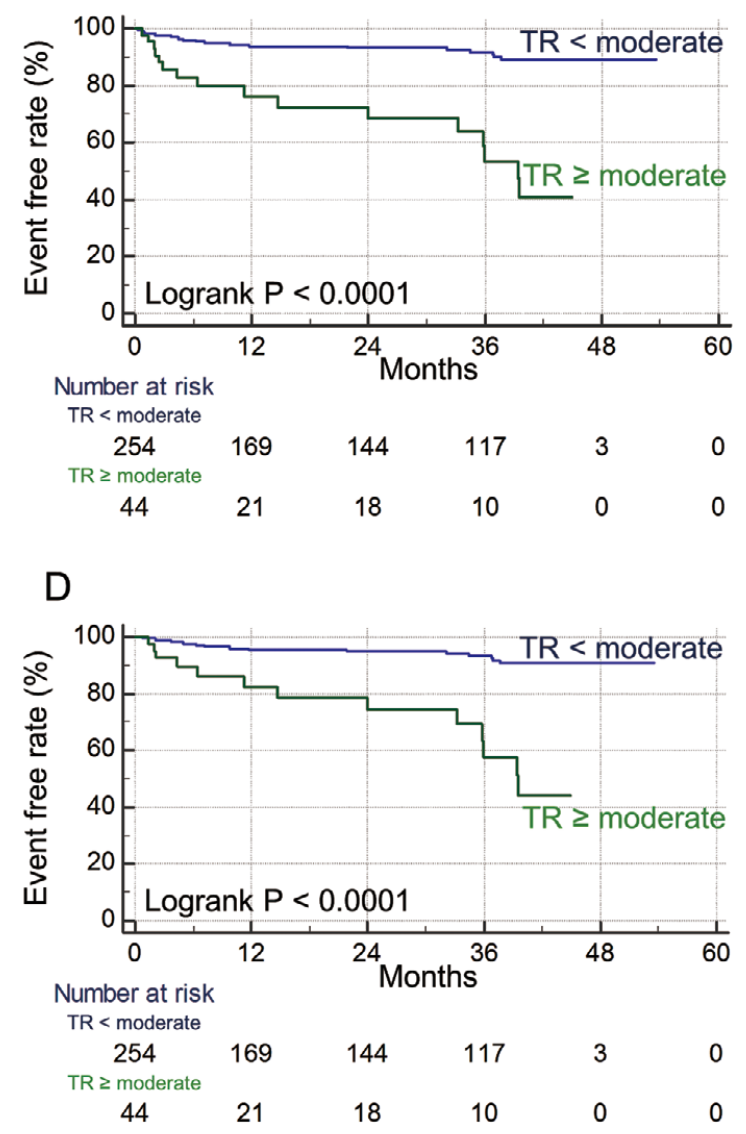

Figure 4. Kaplan-Meier event-free rates of $(\mathbf{A}, \mathbf{B})$ cardiac death, hospitalization due to worsening heart failure, or mitral valve and/ or tricuspid valve repair or (C,D) cardiac death, hospitalization due to worsening heart failure (i.e., excluding valve repair) vs. the presence of significant (i.e., moderate or greater) mitral or tricuspid regurgitations (MR or TR). 
A

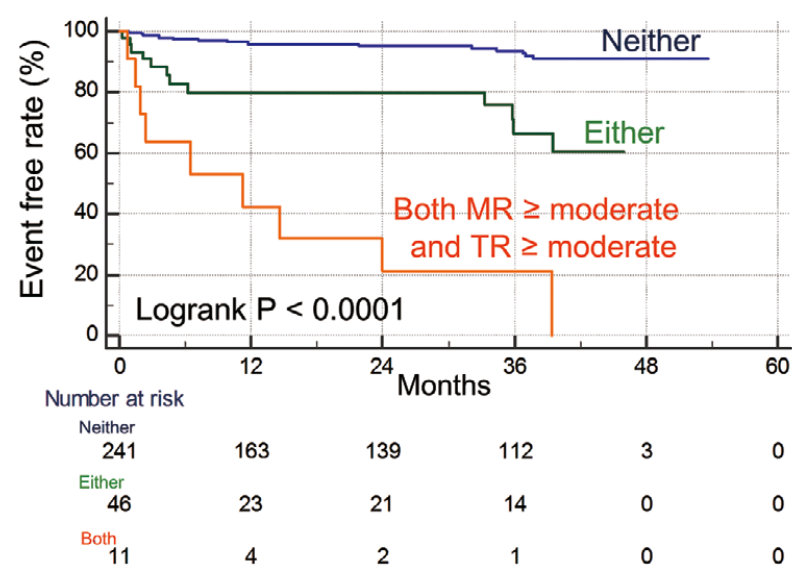

B

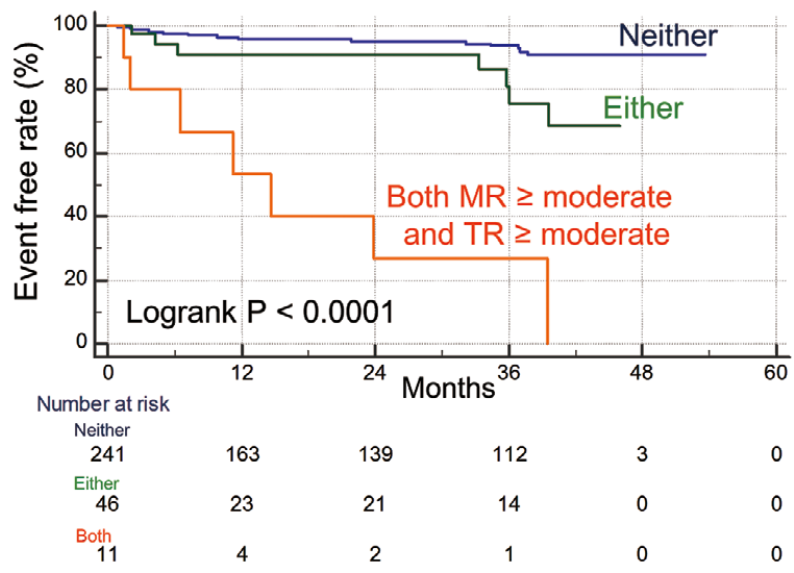

Figure 5. Kaplan-Meier event-free rates of $(\mathbf{A})$ cardiac death, hospitalization due to worsening heart failure, or mitral valve and/ or tricuspid valve repair or (B) cardiac death, hospitalization due to worsening heart failure (i.e., excluding valve repair) vs. the combinations of significant (i.e., moderate or greater) mitral and tricuspid regurgitations (MR and TR).

rate was $69 \%$ with the standard error of $8 \%$ vs. $93 \%$ with the standard error of $2 \%$, respectively, at mean follow-up of 24 months ( $\log$ rank $\mathrm{P}<0.0001$; Figure 4B). We excluded mitral and tricuspid valve repairs from the endpoint to avoid possible intentional bias in surgical indication, but prognosis was still poorer for patients with significant functional MR than without significant functional MR: the event-free rate was $53 \%$ with the standard error of $13 \%$ vs. $95 \%$ with the standard error of $2 \%$, respectively, at mean follow-up of 24 months (log rank $\mathrm{P}<0.0001$; Figure 4C). As well, prognosis was poorer for the patients with significant functional TR than without significant functional TR, even after the exclusion of surgical valve repairs from the endpoint: the event-free rate was $74 \%$ with the standard error of $8 \%$ vs. $95 \%$ with the standard error of $2 \%$, respectively, at mean follow-up of 24 months (log rank $\mathrm{P}<0.0001$; Figure 4D).

In addition, the patients with both significant functional MR and TR had the poorest prognosis, with an event-free rate of $21 \%$ and a standard error of $13 \%$ at mean follow-up of 24 months (Figure 5A). Even after we excluded the surgical repairs from the endpoint, the event-free rate at mean follow-up of 24 months was still low in patients with both significant MR and significant TR $(27 \%$ with the standard error of 16\%; Figure 5B).

\section{Discussion}

The present study has described the prevalence and prognostic significance of functional $\mathrm{MR}$ and $\mathrm{TR}$ in $\mathrm{AF}$ patients despite having preserved LVEF. Overall, (1) functional MR and TR were rarely seen in patients with AF duration $\leq 1$ year; (2) functional MR and TR were seen in one-fourth of patients with longstanding AF > 10 years; (3) both MR and TR were independent predictors of the primary endpoint defined as cardiac death, admission due to worsening HF, or surgical mitral and tricuspid repair; and (4) the concomitance of MR and TR carried the worst prognosis, with an event-free rate of only $21 \%$ at the mean follow-up of 2 years.

Annular dilatation in lone AF patients does not usually cause functional MR, but functional TR is relatively easily induced. ${ }^{25,26}$ In contrast, some other studies have shown that functional MR can occur in patients with AF and an enlarged LA, despite having preserved LV systolic function; this MR is known as atrial functional MR. ${ }^{\mathbf{1 0 - 1 3 , 1 5 , 1 6}}$ In our previous study using 3-D transesophageal echocardiography (TEE), AF patients with atrial functional MR had dilated LA, dilated mitral annulus, flattened anterior mitral leaflet along the mitral annular plane, and posterior mitral leaflet bending toward the LV cavity, traditionally referred as the "hamstringing" phenomenon of the posterior mitral leaflet. ${ }^{13}$ Utsunomiya et al also used 3-D TEE and showed that AF patients with atrial functional TR had dilated RA and tricuspid annulus without valvular tenting. ${ }^{14}$ The present study has shown that the prevalence of atrial functional MR and TR depends on AF duration, which may relate to the degree of LA and RA dilatation. The differences in the prevalence of atrial functional MR and TR between the previous studies and the present study might result from differences in AF duration between the various cohorts. We suppose that the prevalence of functional MR and TR is growing due to the increased number of senior patients with longstanding AF in today's aging population. ${ }^{1-3}$ According to some previous studies, however, regurgitation-induced secondary ventricular dilatation or the lack of leaflet remodeling can be added to other known factors (i.e., AF duration, atrial dilatation, and annular dilatation) as possible causes of the occurrence or the worsening of atrial functional MR and TR..$^{13,27}$ The exact determinants of atrial functional $\mathrm{MR}$ and $\mathrm{TR}$ remain uncertain, therefore further studies are needed to address this issue.

AF can lead to HF with preserved LVEF, and their association brings a poor prognosis. ${ }^{7}$ In fact, cardiac death is more frequent than stroke-related death in AF patients, and a substantial proportion of the cardiac death results from HF. ${ }^{46}$ Consequently, interventions beyond antico- 
agulation are needed to further reduce mortality in AF. The present study confirmed the high probability of future $\mathrm{HF}$ events in AF patients who had both significant functional MR and significant functional TR, thereby suggesting that more intensive therapy may be required for these regurgitations to prevent $\mathrm{HF}$ events in $\mathrm{AF}$ patients. Gertz et al showed that atrial functional MR improved if the sinus rhythm was restored by AF ablation. ${ }^{11}$ The initial LA size, however, was not particularly large in their patients who had significant atrial functional MR: the LA dimension was $4.4 \pm 0.6 \mathrm{~cm}$. Our previous study showed that surgical mitral annuloplasty with concomitant tricuspid annuloplasty may be an effective treatment strategy for reducing MR, HF symptoms, LA size, and HF admissions in patients with a significant degree of atrial functional MR with more dilated LA: the initial LA dimension was $5.2 \pm 0.9 \mathrm{~cm} .{ }^{12}$ Surgical intervention for atrial functional MR and TR could be the best option for HF issues in AF patients with dilated atriums with preserved LVEF.

\section{Study Limitations}

The present study has several limitations. It was a retrospective study based on the medical records of Osaka City General Hospital, and the echocardiography laboratory database. Consequently, MR and TR were graded using various qualification or quantification methods selected at the sonographer's discretion, therefore grading might not be consistent. In our opinion, however, this may be a strength of the present study, rather than a limitation, because these data represent real-world clinical data. In the clinical setting, discrepancy in the grading of MR and TR in AF patients can easily arise with method-by-method and beat-by-beat assessments. Accordingly, we usually use a multiparametric approach with multiple qualification and quantification methods for assessing MR and TR in AF patients, and we finally grade the regurgitations from a comprehensive standpoint under the careful consideration of the advantages and limitations of each method, as done in the present study. We avoided inaccuracy as much as possible by carefully selecting 1 beat occurring after 2 serial beats with average RR interval for the measurement of the systolic parameters. ${ }^{20}$ In addition, we used 3 levels of borderline grades (none-mild, mild-moderate, and moderatesevere) when a considerable discrepancy existed in the grading between the multiple methods or between the different beats.

A further challenge is obtaining an accurate evaluation of LV diastolic dysfunction as a possible cause of HF with preserved LVEF in AF patients. ${ }^{28}$ We did not assess LV diastolic parameters from the calculation of the average at multiple beats; instead, we used the measurements at a single representative beat, although the single-beat measurement is not recommended in the assessment of diastolic parameters in AF patients. ${ }^{29}$ Consequently, the present single-beat measurements might disturb an accurate evaluation of the relationship between diastolic parameters and HF events. Future prospective studies using the recommended methods for echocardiographic measurements are needed to address this issue.

\section{Conclusions}

Significant functional MR and TR were seen in a substantial proportion of patients with longstanding AF despite having preserved LVEF. The combination of these regurgitations, termed "atrial functional MR and TR", is associated with poor prognosis in AF patients with preserved LVEF and may have to be treated more intensively.

\section{Disclosures}

The authors declare no conflicts of interest.

\section{References}

1. Chugh SS, Havmoeller R, Narayanan K, Singh D, Rienstra M, Benjamin EJ, et al. Worldwide epidemiology of atrial fibrillation: A Global Burden of Disease 2010 Study. Circulation 2014; 129: 837-847.

2. Krijthe BP, Kunst A, Benjamin EJ, Lip GY, Franco $\mathrm{OH}$, Hofman A, et al. Projections on the number of individuals with atrial fibrillation in the European Union, from 2000 to 2060. Eur Heart J 2013; 34: 2746-2751.

3. Benjamin EJ, Blaha MJ, Chiuve SE, Cushman M, Das SR, Deo $\mathrm{R}$, et al. Heart disease and stroke statistics-2017 update: A report from the American Heart Association. Circulation 2017; 135: e146-e603.

4. Marijon E, Le Heuzey JY, Connolly S, Yang S, Pogue J, Brueckmann $\mathrm{M}$, et al. Causes of death and influencing factors in patients with atrial fibrillation: A competing-risk analysis from the randomized evaluation of long-term anticoagulant therapy study. Circulation 2013; 128: 2192-2201.

5. Pokorney SD, Piccini JP, Stevens SR, Patel MR, Pieper KS, Halperin JL, et al. Cause of death and predictors of all-cause mortality in anticoagulated patients with nonvalvular atrial fibrillation: Data from ROCKET AF. J Am Heart Assoc 2016; 5: $\mathrm{e} 002197$.

6. Gómez-Outes A, Lagunar-Ruíz J, Terleira-Fernández AI, CalvoRojas G, Suárez-Gea ML, Vargas-Castrillón E. Causes of death in anticoagulated patients with atrial fibrillation. $\mathrm{J} \mathrm{Am}$ Coll Cardiol 2016; 68: 2508-2521.

7. Zakeri R, Chamberlain AM, Roger VL, Redfield MM. Temporal relationship and prognostic significance of atrial fibrillation in heart failure patients with preserved ejection fraction: A community-based study. Circulation 2013; 128: 1085-1093.

8. Ho JE, Gona P, Pencina MJ, Tu JV, Austin PC, Vasan RS, et al. Discriminating clinical features of heart failure with preserved vs. reduced ejection fraction in the community. Eur Heart $J$ 2012; 33: $1734-1741$.

9. Wang TJ, Larson MG, Levy D, Vasan RS, Leip EP, Wolf PA, et al. Temporal relations of atrial fibrillation and congestive heart failure and their joint influence on mortality: The Framingham Heart Study. Circulation 2003; 107: 2920-2925.

10. Kihara T, Gillinov AM, Takasaki K, Fukuda S, Song JM, Shiota M, et al. Mitral regurgitation associated with mitral annular dilation in patients with lone atrial fibrillation: An echocardiographic study. Echocardiography 2009; 26: 885-889.

11. Gertz ZM, Raina A, Saghy L, Gertz ZM, Raina A, Saghy L. Evidence of atrial functional mitral regurgitation due to atrial fibrillation: Reversal with arrhythmia control. J Am Coll Cardiol 2011; 58: 1474-1481.

12. Takahashi Y, Abe Y, Sasaki Y, Bito Y, Morisaki A, Nishimura S, et al. Mitral valve repair for atrial functional mitral regurgitation in patients with chronic atrial fibrillation. Interact Cardiovasc Thorac Surg 2015; 21: 163-168.

13. Ito $\mathrm{K}$, Abe Y, Takahashi Y, Shimada Y, Fukumoto H, Matsumura $Y$, et al. Mechanism of atrial functional mitral regurgitation in patients with atrial fibrillation: A study using three-dimensional transesophageal echocardiography. $J$ Cardiol 2017; 70: 584-590.

14. Utsunomiya H, Itabashi Y, Mihara H, Berdejo J, Kobayashi S, Siegel RJ, et al. Functional tricuspid regurgitation caused by chronic atrial fibrillation: A real-time 3-dimensional transesophageal echocardiography study. Circ Cardiovasc Imaging 2017; 10: e004897.

15. Machino-Ohtsuka T, Seo Y, Ishizu T, Sato K, Sugano A, Yamamoto $M$, et al. Novel mechanistic insights into atrial functional mitral regurgitation: 3-dimensional echocardiographic study. Circ J 2016; 80: 2240-2248.

16. Yamano T, Furukawa K, Matoba S. "Hamstring leaflet" now demonstrated to be a determinant of functional mitral regurgitation in patients with atrial fibrillation. Circ J 2016; 80: 2098-2099.

17. Joint Task Force on the Management of Valvular Heart Disease 
of the European Society of Cardiology (ESC); European Association for Cardio-Thoracic Surgery (EACTS), Vahanian A, Alfieri O, Andreotti F, Antunes MJ, Barón-Esquivias G, Baumgartner $\mathrm{H}$, et al. Guidelines on the management of valvular heart disease (version 2012). Eur Heart J 2012; 33: 2451 - 2496.

18. Nishimura RA, Otto CM, Bonow RO, Carabello BA, Erwin JP 3rd, Guyton RA, et al. 2014 AHA/ACC guideline for the management of patients with valvular heart disease: A report of the American College of Cardiology/American Heart Association Task Force on Practice Guidelines. Circulation 2014; 129: 2440-2492. Erratum in: Circulation 2014; 129: e650.

19. Ryan T, Armstrong WF, Khandheria BK; American Society of Echocardiography. Task Force 4: Training in echocardiography endorsed by the American Society of Echocardiography. $J$ Am Coll Cardiol 2008; 51: $361-367$

20. Sumida T, Tanabe K, Yagi T, Kawai J, Konda T, Fujii Y, et al. Single-beat determination of Doppler-derived aortic flow measurement in patients with atrial fibrillation. J Am Soc Echocardiogr 2003; 16: 712-715.

21. Lang RM, Bierig M, Devereux RB, Flachskampf FA, Foster E, Pellikka PA, et al. Recommendations for chamber quantification: A report from the American Society of Echocardiography's Guidelines and Standards Committee and the Chamber Quantification Writing Group, developed in conjunction with the European Association of Echocardiography, a branch of the European Society of Cardiology. J Am Soc Echocardiogr 2005; 18: $1440-1463$.

22. Zoghbi WA, Enriquez-Sarano M, Foster E, Grayburn PA, Kraft $\mathrm{CD}$, Levine RA, et al. Recommendations for evaluation of the severity of native valvular regurgitation with two-dimensional and Doppler echocardiography. J Am Soc Echocardiogr 2003; 16: $777-802$.

23. Rudski LG, Lai WW, Afilalo J, Rudski LG, Lai WW, Afilalo J.
Guidelines for the echocardiographic assessment of the right heart in adults: A report from the American Society of Echocardiography endorsed by the European Association of Echocardiography, a registered branch of the European Society of Cardiology, and the Canadian Society of Echocardiography. $J$ Am Soc Echocardiogr 2010; 23: 685-713, quiz 786-788.

24. Nagueh SF, Appleton CP, Gillebert TC, Marino PN, Oh JK, Smiseth OA, et al. Recommendations for the evaluation of left ventricular diastolic function by echocardiography. $J$ Am Soc Echocardiogr 2009; 22: 107-133.

25. Otsuji Y, Kumanohoso T, Yoshifuku S, Matsukida K, Koriyama $\mathrm{C}$, Kisanuki A, et al. Isolated annular dilation does not usually cause important functional mitral regurgitation: Comparison between patients with lone atrial fibrillation and those with idiopathic or ischemic cardiomyopathy. J Am Coll Cardiol 2002; 39: $1651-1656$.

26. Zhou X, Otsuji Y, Yoshifuku S, Zhou X, Otsuji Y, Yoshifuku S. Impact of atrial fibrillation on tricuspid and mitral annular dilatation and valvular regurgitation. Circ J 2002; 66: 913-916.

27. Kagiyama N, Hayashida A, Toki M, Fukuda S, Ohara M, Hirohata A, et al. Insufficient leaflet remodeling in patients with atrial fibrillation: Association with the severity of mitral regurgitation. Circ Cardiovasc Imaging 2017; 10: e005451.

28. Okura H, Takada Y, Kubo T, Iwata K, Mizoguchi S, Taguchi $\mathrm{H}$, et al. Tissue Doppler-derived index of left ventricular filling pressure, E/E', predicts survival of patients with non-valvular atrial fibrillation. Heart 2006; 92: 1248- 1252.

29. Kusunose K, Yamada H, Nishio S, Tomita N, Niki T, Yamaguchi K, et al. Clinical utility of single-beat E/e' obtained by simultaneous recording of flow and tissue Doppler velocities in atrial fibrillation with preserved systolic function. $J A C C$ Cardiovasc Imaging 2009; 2: 1147-1156. 